\title{
Repeatability of 3D OCT Pachymetry in Eyes with and without Contact Lens Induced Corneal Swelling
}

\author{
Victoria de Juan', Ana B. Cisneros ${ }^{2}$, Raul Martin ${ }^{1,3}$ \\ ${ }^{1}$ Optometry Research Group, IOBA-Eye Institute, University of Valladolid, Valladolid, Spain \\ ${ }^{2}$ Cervantes Eye Clinic, Valladolid, Spain \\ ${ }^{3}$ Department of Physics \& Optics, School of Optometry, University of Valladolid, Valladolid, Spain \\ Email: victoria@ioba.med.uva.es
}

Received December 1, 2012; revised January 5, 2013; accepted January 14, 2013

Copyright (C) 2013 Victoria de Juan et al. This is an open access article distributed under the Creative Commons Attribution License, which permits unrestricted use, distribution, and reproduction in any medium, provided the original work is properly cited.

\begin{abstract}
Introduction: Corneal thickness measurement is an indirect assessment of the physiological corneal function in human eye. This value, especially in the central area, is an important data for clinical diagnosis, treatment and monitoring of anterior segment pathologies. There are several technologies for non-invasively measuring corneal thickness like optical coherence tomography (OCT). However, there is little information available describing the effect of contact lens induced corneal swelling in OCT measures. The purpose of this study was to evaluate the repeatability of OCT corneal pachymetry in eyes with and without contact lens induced corneal swelling. Material and Methods: This study included five visits over one week in six healthy subjects: baseline and after sleeping with four different CL of $+0.50 \mathrm{D}$, $+2.00 \mathrm{D},+5.00 \mathrm{D}$ and $+8.00 \mathrm{D}$ (Acuvue 2, Johnson \& Johnson Vision Care) on four different days. Corneal pachymetry OCT 3D (Topcon) were measured three consecutive times during each visit. Besides, this measurement was repeated in 12 meridians (Scans \#1 to \#12), between $0^{\circ}$ and $165^{\circ}$ with $15^{\circ}$ intervals. Results: Corneal pachymetry was significant different between before and after contact lens wear $(p<0.001$ ANOVA). High repeatability was found without corneal swelling [Coefficient of variation $(\mathrm{CV})=0.68 \% p=0.93$ ] and with corneal swelling [CL $-0.50 \mathrm{D}(\mathrm{CV}=0.78 \% p=$ $0.95 ; 590 \pm 46 \mu \mathrm{m} \mathrm{CI} 95 \% 548$ to 596), with CL $+2.00 \mathrm{D}(\mathrm{CV}=0.72 \% p=0.97 ; 601 \pm 46 \mu \mathrm{m}$ CI $95 \% 595$ to 607$)$, with $\mathrm{CL}+5.00 \mathrm{D}(\mathrm{CV}=0.78 \% p=0.66 ; 608 \pm 50 \mu \mathrm{m} \mathrm{CI} 95 \% 601$ to 615$)$ and with $\mathrm{CL}+8.00 \mathrm{D}(\mathrm{CV}=0.77 \% p=$ $0.97 ; 607 \pm 44 \mu \mathrm{m} \mathrm{CI} \mathrm{95 \%} 601$ to 613$)]$. There were no differences $(p=1.00)$ in central corneal thickness along the 12 scans corneal [Scan \#0 (593 $\pm 50 \mu \mathrm{m}$ CI 95\% 582 to 603), \#1 (592 $\pm 50 \mu \mathrm{m}$ CI 95\% 581 to 603), \#2 (591 $\pm 50 \mu \mathrm{m}$ IC 95\% 580 to 602), \#3 (590 $\pm 49 \mu \mathrm{m} \mathrm{CI} \mathrm{95 \%} 579$ to 600), \#4 (590 $\pm 50 \mu \mathrm{m}$ CI 95\% 579 to 600), \#5 (591 $\pm 50 \mu \mathrm{m} \mathrm{CI} \mathrm{95 \%}$

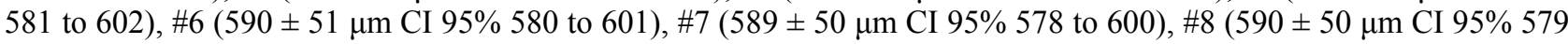
to 600$)$, \#9 (589 $\pm 50 \mu \mathrm{m}$ CI $95 \% 579$ to 600$)$, \#10 (592 $\pm 50 \mu \mathrm{m} \mathrm{CI} \mathrm{95 \%} 581$ to 602) and \#11 (591 $\pm 50 \mu \mathrm{m} \mathrm{CI} 95 \%$ 580 to 601$)$. Conclusions: 3D OCT showed a high repeatability $(\mathrm{CV}<1 \%)$ to measure central thickness in corneas with and without contact lens induced corneal swelling. This technology presents advantages over other clinical devices used to measure corneal thickness and could be the "gold standard" for future clinical studies and clinical practice for eye care practitioners.
\end{abstract}

Keywords: Optical Coherence Tomography; Corneal Swelling; Contact Lenses; Corneal Pachymetry

\section{Introduction}

Corneal thickness is an indirect measurement of the physiological function of cornea, so the pachymetry, especially in the central area, is important for diagnosis, treatment and monitoring some eye pathologies of the anterior segment [1]. Central corneal thickness measurement has become increasingly important in the assessment of a variety of corneal diseases, the precision of intraocular pressure readings in glaucoma diagnosis and management, and eligibility of patients considering re- fractive surgery [2] and others. Furthermore, changes in corneal thickness can be a warning sign of endothelial cell loss, which can be helpful in evaluating the safety of iris-fixated phakic intraocular lenses or intraocular surgery for example in cataract surgery [3].

On the other hand, contact lenses (CL) wear is a common way to correct eye refractive errors like myopia, hyperopia or astigmatism with millions of wearers in the world. However, CL wear is associated with adverse ocular responses, most of them related with corneal 
swelling, when the cornea is subjected to hypoxia [4]. Usually, corneal swelling is calculated with the increase of corneal thickness with precise and effective methods, such as optical coherence tomography (OCT) [5].

Besides, corneal swelling is directly related to the oxygen transmissibility $(\mathrm{Dk} / \mathrm{t})$ of CLs, and it is a quantitative measurement for assessing the impact of corneal hypoxia of CLs during wear. In fact, it has been shown that there is a direct relationship between the central thickness and corneal edema [6].

OCT is a medical imaging technology that generates high-resolution cross-sectional images of subsurface internal tissue structure in situ and in real time, with a wide variety of applications in medicine. Due to the transparent ocular structures, the main field of OCT applications is the Ophthalmology and OCT was first introduced in 1991 to image the posterior segment of the eye [7] and three years later, in 1994, the first report of anterior segment OCT imaging was reported [8]. Since then, and due to the successful diffusion of the refractive surgery, the refinement of this technique has been permanently progressing for the anterior eye analysis.

There are currently two commercial different types of OCT in ophthalmic practice: time-domain OCT, where the cross-sectional images are produced by varying the position of the reference mirror; and Fourier-domain OCT (primarily developed to assess the posterior segment), where the reference mirror is fixed and the crosssectional images are obtain by Fourier transformation of the spectral interferogram (interference between the sample and the reference reflections) [9].

Fourier-domain systems such as the 3D OCT (Topcon Medical Systems Inc., Paramus, NJ, USA), the Slit Lamp-OCT (Heidelberg Engineering GmbH, Heidelberg, Germany) or the RTVue (Optovue Inc., Fremont, California) provide a faster acquisition than time-domain systems, such as the AS-OCT Visante (Carl Zeiss Meditec Inc., Dublin, CA, USA). In addition, its higher resolution allows the visualization of more details, although time-domain systems shows all anterior segments structures in a single image, while Fourier-domain shows just a little part of a single component of the anterior segment of the eye.

Moreover, OCT allows a wide variety of uses for the anterior segment examination in postoperative eyes. Especially, in refractive surgery procedures, for example in post-LASIK patients is useful to evaluate the flap and the residual stromal bed thickness before a retreatment procedure in order to avoid a post-LASIK ectasia.

One of the major advantages of the OCT systems is that it allows non-invasive measurement of the corneal thickness without CL removing, which makes it an excellent tool to monitor corneal thickness changes during CL wear $[5,10]$.
There are several technologies to non-invasive measurement of corneal thickness such as OCT, Orbscan II and Pentacam. Besides, these non-contact methods eliminate the disadvantage of the ultrasonic pachymetry, with the risk of corneal abrasion and infections due to corneal contact with the probe and discomfort for the patient.

However, there is little information available describing the effect of $\mathrm{CL}$ induced corneal swelling in OCT pachymetry measurement, so the purpose of this study was to evaluate the repeatability of 3D OCT corneal pachymetry in eyes without and with different level of $\mathrm{CL}$ induced corneal swelling.

\section{Material and Methods}

\subsection{Subjects}

Six subjects participated in the study. Three were women and three men (mean age, $27.17 \pm 4.1$ years; range, 23 34). The refractive error in terms of spherical equivalent ranged from +0.50 to $-3.00 \mathrm{D}(-1.58 \pm 1.49 \mathrm{D})$. Subjects were excluded if they had history of ocular surgery, an active ocular surface disease, such as significant dry eye, papillary conjunctivitis, corneal opacities, current medication that could affect ocular physiology, or if they had previously worn extended CL wear. Subjects reported monocular best spectacle-corrected visual acuity of 20/20 or better.

Informed consent was obtained from each subject after approval was granted by the Human Sciences Ethics Committee of the University of Valladolid. All subjects were treated in accordance with the Declaration of Helsinki.

\subsection{Instrumentation}

Corneal thickness was measured with 3D OCT-2000, (Topcon, Japan). Three consecutive measurements (\#1, \#2 and \#3) were taken on each cornea during each study visit. The mean of the three scans of each cornea was used as the final result to corneal swelling calculation. The same experimented and masked operator performed all OCT scans during all visits.

The measurement of corneal thickness in the corneal apex was assessed with the tool "caliper" (Figure 1) of the OCT software. This measurement was repeated in all 12 scanned meridians, between $0^{\circ}$ and $165^{\circ}$ with $15^{\circ}$ intervals.

\subsection{Procedures}

The study was a 1-week, prospective, controlled trial. A CL was randomly fitted in one eye and the fellow eye was used as a control eye. An independent investigator composed the randomized schedule.

This study included five visits over one week: baseline 


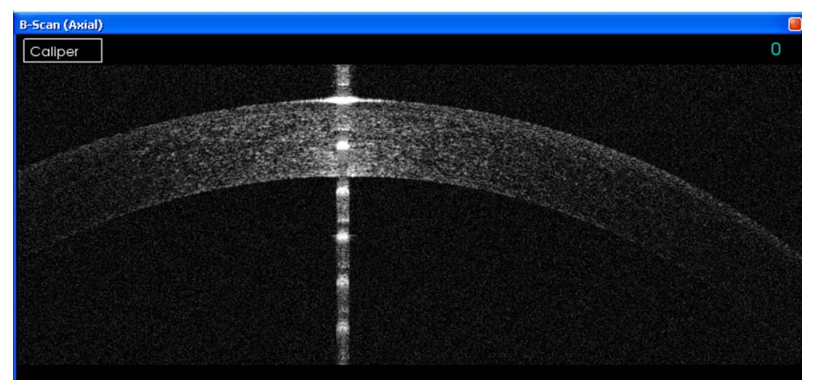

Figure 1. Screen capture of OCT 3D measurements.

and after sleeping with four different CL of $+0.50 \mathrm{D}$, $+2.00 \mathrm{D},+5.00 \mathrm{D}$ and +8.00 D (Acuvue 2, Etafilcon-A material, Johnson \& Johnson Vision Care, USA) on four different days. Etafilcon-A is a 7 days' extended wear (EW) approved hydrogel lens in the United States. The difference in power of the CL induces different levels of hypoxia and thus various degrees of corneal swelling.

During the baseline visit (day 0), all participants were screened according to inclusion criteria and pachymetry with OCT was performed. All baseline measurements were performed in the afternoon, between 4 p.m. and 8 p.m., because some authors suggested that this is the time of the day when the eye is physiologically more stable [11]. All follow-up visits were early in the morning and the eye with the CL was occluded while it was worn, to ensure that induced overnight corneal swelling was present at the time of the pachymetry measurement.

\subsection{Statistical Analysis}

Statistical analysis was performed using the Statistical Package for the Social Sciences (SPSS for Windows software, version 15.0, SPSS, Inc., Chicago, IL, USA). Descriptive statistics included mean, standard deviation (SD), and $95 \%$ of confidence interval (CI 95\%), were calculated.

Repeatability was assessed with coefficient of variation (CV) and Bland-Altman analysis (mean difference and limits of agreement). Repeated measures analysis of variance (ANOVA) was used to compare the measurements of the every different scan. $P$ value less than 0.05 was considered statistically significant.

\section{Results}

None of the subjects had significant biomicroscopic signs (grade $>1$ in Efron grading scale [12] of CL complications (corneal staining, limbal injection, striae, folds, or others).

\subsection{Corneal Swelling}

We found an increase of corneal thickness in all eyes after wearing CL. Comparing with control eyes significant statistical differences ( $p<0.001$ ANOVA) were found between both groups. Eyes who wear CL with lower $\mathrm{Dk} / \mathrm{t}$, due to $\mathrm{CL}$ thick thickness (more positive power), showed higher corneal swelling, although not significant statistical differences were found $(p=0.614$ ANOVA).

\subsection{Repeatability of Pachymetry}

At baseline visit, (without CL), there were no statistical significant differences between measurements \#1, \#2 and \#3 (ANOVA, $p=0.925$ ). Alike, with every CL used no statistical differences $(p>0.662)$ were found between repeated measurements (Table 1 and Figure 2).

\subsection{Differences in Pachymetry after Wearing CL with Different Power}

There were statistical significant differences between corneal pachymetry with and without CL worn (ANOVA $p<0.01)$. CLs with plus power $(+2.00 \mathrm{D},+5.00 \mathrm{D}$ and $+8.00 \mathrm{D})$ showed greater amount of corneal swelling than $+0.50 \mathrm{D}$ CL. There was statistical significant different between +0.50 D CL and +5.00 D CL $(p=0.002$ ANOVA with Bonferroni correction). Also, there was a difference between $+0.50 \mathrm{D} \mathrm{CL}$ and $+8.00 \mathrm{D} \mathrm{CL}(p=$ 0.001 ANOVA with Bonferroni correction) Figure 2.

\subsection{Repeatability of Pachymetry of Different OCT Scans in Different Corneal Meridians}

Table 2 shows descriptive analysis of pachymetry measurements at 12 different meridians without statistical significant ( $p>0.05$ ANOVA) differences among them.

\section{Discussion}

OCT is a rapid, reproducible, non-invasive method of imaging of the anterior eye segment, capable of assess repeated cross-sectional measurements of the central part of the cornea. Moreover, OCT has shown excellent reproducibility for central corneal thickness and anterior chamber depth measurements in healthy eyes [13].

Agarwal and coauthors used automated method of anterior segment OCT in eyes with decreased corneal clarity and compromised anterior chamber visualization attributable to corneal edema [14]. In some cases, OCT is used to evaluate the change in thickness of the anterior, stromal and posterior corneal structures in response to hypoxia-induced corneal swelling with good results [6].

However, OCT pachymetry could not to be interchangeable with other devices because significant differences were found between OCT, ultrasound pachymeter and Orbscan II pachymetry in corneal thickness measurements in healthy corneas $[2,15]$ and in patients after LASIK refractive surgery [16].

On the other hand, central corneal thickness and ante- 
Table 1. Descriptive statistics of pachymetric measures with 3D OCT. The mean of three repeated measurements was taken as summary value for each study eye.

\begin{tabular}{|c|c|c|c|c|c|}
\hline & Mean \pm SD (CI 95\%) $\mu \mathrm{m}$ & Repeatability ANOVA (p) & CV (\%) & Mean difference \pm DS $(\mu \mathrm{m})$ & Limits of agreement $(\mu \mathrm{m})$ \\
\hline Baseline & $\begin{array}{c}548.15 \pm 37.08 \\
(543.18 \text { to } 553.13)\end{array}$ & 0.925 & 0.68 & $-0.15 \pm 4.08$ & 7.85 to -8.15 \\
\hline$-0.50 \mathrm{D} \mathrm{CL}$ & $\begin{array}{c}590.31 \pm 46.11 \\
(548.11 \text { to } 596.51)\end{array}$ & 0.951 & 0.78 & $-0.08 \pm 4.59$ & 8.92 to -9.08 \\
\hline$+2.00 \mathrm{D} \mathrm{CL}$ & $\begin{array}{c}600.88 \pm 45.53 \\
(594.78 \text { to } 606.99)\end{array}$ & 0.966 & 0.71 & $-0.05 \pm 4.16$ & 8.11 to -8.21 \\
\hline$+5.00 \mathrm{D} \mathrm{CL}$ & $\begin{array}{c}607.99 \pm 49.97 \\
(601.09 \text { to } 614.88)\end{array}$ & 0.662 & 0.78 & $-0.10 \pm 4.36$ & 8.46 to -8.66 \\
\hline$+8.00 \mathrm{D} \mathrm{CL}$ & $\begin{array}{c}607.00 \pm 44.02 \\
(601.07 \text { to } 612.94)\end{array}$ & 0.966 & 0.76 & $0.02 \pm 4.66$ & 9.15 to -9.11 \\
\hline
\end{tabular}

Table 2. Descriptive analysis of corneal pachymetry at 12 different OCT scans in different corneal meridians.

\begin{tabular}{cccccc}
\hline & Mean \pm SD & CI 95\% & & Mean \pm SD & CI 95\% \\
\hline $\mathbf{0}^{\circ}$ Scan & $592.58 \pm 50.07$ & $582.04-603.13$ & $\mathbf{9 0}^{\circ}$ Scan & $590.19 \pm 50.62$ & $579.53-600.86$ \\
$\mathbf{1 5}^{\circ}$ Scan & $592.07 \pm 50.37$ & $581.46-602.68$ & $\mathbf{1 0 5}^{\circ}$ Scan & $589.10 \pm 50.30$ & $578.50-599.70$ \\
$\mathbf{3 0}^{\circ}$ Scan & $591.00 \pm 50.19$ & $580.43-601.57$ & $\mathbf{1 2 0}^{\circ}$ Scan & $589.81 \pm 50.44$ & $579.18-600.43$ \\
$\mathbf{4 5}^{\circ}$ Scan & $589.76 \pm 49.25$ & $579.26-600.26$ & $\mathbf{1 3 5}^{\circ}$ Scan & $589.44 \pm 50.03$ & $578.90-599.98$ \\
$\mathbf{6 0}^{\circ}$ Scan & $589.82 \pm 49.66$ & $579.29-600.34$ & $\mathbf{1 5 0}^{\circ}$ Scan & $591.83 \pm 50.19$ & $581.26-602.40$ \\
$\mathbf{7 5}^{\circ}$ Scan & $591.24 \pm 50.24$ & $580.65-601.82$ & $\mathbf{1 6 5}^{\circ}$ Scan & $590.87 \pm 50.08$ & $580.31-601.42$ \\
\hline
\end{tabular}

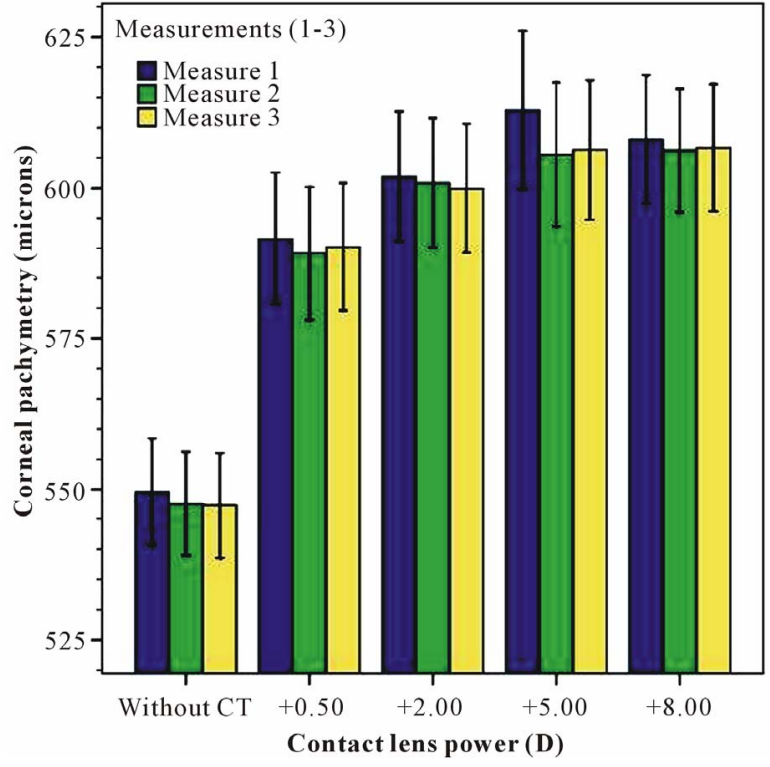

Figure 2. Summary of central corneal thickness before and after each CL wear.

rior chamber depth measurements using AS-OCT, Orbscan and Pentacam demonstrated high intraobserver reliability. However, these three devices should not be used interchangeably for anterior chamber depth measurement in healthy subject and patients after phakic intraocular lens implantation. ACD measurements demonstrated significant differences among the three instruments [2].

Differences between corneal pachymetry and anterior camber depth could be related with the differences in technology used to capture and image analysis; especially with other image device like slit-lamp topography (Orbscan) of Scheimpflug corneal topography (Pentacam).

In addition, there are not studies focused over the effect of corneal swelling in central corneal thickness measured with OCT. Corneal swelling increase corneal hydration and light scattering, but in the range of clinically accepted CL wear, the effect of swelling have not show any significant effect over the OCT 3D pachymetry measurements.

Our data demonstrate minimal differences in corneas with and without corneal swelling. This study revealed that 3D OCT device showed a high repeatability $(\mathrm{CV}<$ $1 \%$ ) in corneas with and without CL induced corneal swelling. The repeated measurements (\#1, \#2 and \#3) have not shown significant differences along the study visits, with different degree of corneal swelling induced by different oxygen transmissibility CL worn. Power differences in the same type of CL increase central lens thickness and reduce the oxygen transmissibility through the CL $[6,17]$. However, major limitation of the study is the small sample size, so these results may be interpreted with caution.

This information could be useful to determine the effect of different CL wear over corneal physiology and non-invasively control of the changes induced by $\mathrm{CL}$ wear, introducing the OCT measurements in clinical 
practice and research in anterior eye and CL practice, because the main application of the OCT in eye assessment was focused in posterior eye analysis (retina) [10].

The applicability of the results of this study could be immediate in eye care practice and very valuable in CL practice, because it would improve eye care services in a large numbers of patients. CL induced corneal swelling is related with many complications $[4,12]$ in these patients, so OCT could measure and monitoring the levels of swelling, and maintaining the corneal swelling in safe levels avoiding the compromise of the corneal physiology.

OCT technology presents several advantages (mainly, non-invasive, accuracy and high repeatability) over other clinical devices or techniques used to measure corneal thickness and could be the "gold standard" for future clinical studies and clinical practice for eye care practitioners.

\section{REFERENCES}

[1] F. W. Price Jr., D. L. Koller and M. O. Price, "Central Corneal Pachymetry in Patients Undergoing Laser in Situ Keratomileusis," Ophtalmology, Vol. 106, No. 11, 1999, pp. 2216-2220.

[2] M. Doors, L. P. Cruysberg, T. T. Berendschot, J. de Brabander, F. Verbaket, C. A. Webers and R. M. Nuijts, "Comparison of Central Corneal Thickness and Anterior Chamber Depth Measurements Using Three Imaging Technologies in Normal Eyes and after Phakic Intraocular Lens Implantation," Graefes Archive for Clinical and Experimental Ophthalmology, Vol. 247, No. 8, 2009, pp. 1139-1146.

[3] H. Cheng, A. K. Bates, L. Wood and K. McPherson, "Positive Correlation of Corneal Thickness and Endothelial Cell Loss. Serial Measurements after Cataract Surgery," Arch Ophtalmology, Vol. 106, No. 7, 1988, pp. 920-922. doi:10.1001/archopht.1988.01060140066026

[4] K. Dumbleton, “Adverse Events with Silicone Hydrogel Continuous Wear," Contact Lens \& Anterior Eye, Vol. 25, No. 3, 2002, pp. 137-146. doi:10.1016/S1367-0484(02)00009-7

[5] R. Martin, V. de Juan, G. Rodriguez, et al., "Measurement of Corneal Swelling Variations without Removal of the Contact Lens during Extended Wear," Investigative Ophthalmology \& Visual Science, Vol. 48, No. 7, 2007, pp. 3043-3050. doi:10.1167/iovs.06-1372

[6] N. Hutchings, T. L. Simpson, C. Hyun, A. A. Moayed, et al., "Swelling of the Human Cornea Revealed by HighSpeed, Ultrahight-Resolution Optical Coherence Tomography," Investigative Ophthalmology \& Visual Science, Vol. 9, No. 51, 2010, pp. 4579-4584.

[7] M. Doors, T. T. Berendschot, J. de Brabander, et al., "Va- lue of Optical Coherence Tomography for Anterior Segment Surgery," Journal of Cataract \& Refractive Surgery, Vol. 36, No. 7, 2010, pp. 1213-1229. doi:10.1016/j.jers.2010.05.002

[8] J. A. Izatt, M. Hee, E. A. Swanson, et al., "MicrometerScale Resolution Imaging of the Anterior Eye in Vivo with Optical Coherence Tomography," Archives of Ophthalmology, Vol. 112, No. 12, 1994, pp. 1584-1589. doi:10.1001/archopht.1994.01090240090031

[9] J. L. B. Ramos, Y. Li and D. Huang, "Clinical and Research Applications of Anterior Segment Optical Coherence Tomography-A Review," Clinical \& Experimental Ophthalmology, Vol. 37, No. 1, 2009, pp. 81-89. doi:10.1111/j.1442-9071.2008.01823.x

[10] A. F. Fercher, W. Drexler, C. K. Hitzenberger and T. Lasser, "Optical Coherence Tomography_Principles and Applications," Reports on Progress in Physics, Vol. 66, No. 2, 2003, pp. 239-303. doi:10.1088/0034-4885/66/2/204

[11] M. J. González-Méijome, A. Cerviño, E. Yebra-Pimentel, et al., "Central and Peripheral Corneal Thickness Measurement with Orbscan II and Topographical Ultrasound Pachymetry," Journal of Cataract \& Refractive Surgery, Vol. 29, No. 1, 2003, pp. 125-132. doi:10.1016/S0886-3350(02)01815-1

[12] N. Efron, "Contact Lens Complications," 2nd Edition, Butterworth-Heinemann, Oxford, 2004.

[13] F. Scholomit, B. A. Sandler, R. Joseph, M. O. Zelefsky, M. D. Syrili Dorairaj, et al., "Intra-observer and InterObserver Reliability and Reproductibility of Slit-LampAdapted Optical Coherence Tomography for Evaluation of Anterior Chamber Depth and Central Corneal Thickness," Ophtalmic Surgery, Lasers \& Imaging, Vol. 39, No. 4, 2008, pp. 299-303.

[14] A. Agarwal, D. Ashokkumar, S. Jacob and Y. Saravaman, "High-Speed Optical Coherence Tomography for Imaging Anterior Chamber Inflammatory Reaction in Uveitis: Clinical Correlation and Grading," American Journal of Ophthalmology, Vol. 147, No. 3, 2009, pp. 413-416. doi:10.1016/j.ajo.2008.09.024

[15] J. Thomas, J. Wang, A. M. Rollins and J. Sturm, “Comparison of Corneal Thickness Measured with Optical Coherence Tomography, Ultrasonic Pachymetry and a Scanning Slit Method," Journal of Refractive Surgery, Vol. 7, No. 22, 2006, pp. 671-678.

[16] R. B. Kucumen, N. H. Yenerel, E. Gorgun, D. Oral, et al, "As OCT as a Tool for Flap Thickness Measurement after Femtosecond-Assisted LASIK," Ophtalmic Surgery, Lasers \& Imaging, Vol. 42, No. 1, 2011, pp. 31-36.

[17] D. M. Harvitt and J. A. Bonanno, "Re-Evaluation of the Oxygen Diffusion Model for Predicting Minimum Contact Lens Dk/t Values Needed to Avoid Corneal Anoxia," Optometry \& Vision Science, Vol. 76, No. 10, 1999, pp. 712-719. 